\title{
Recurrence Relations for Single and Product Moments of Generalized Order Statistics from $p$ th Order Exponential Distribution and its Characterization
}

\author{
Devendra Kumar
}

Aligarh Muslim University

\begin{abstract}
In this paper, we establish some recurrence relations for single and product moments of generalized order statistics from $p$ th order exponential distribution. Further the results are deduced for the recurrence relations of record values and ordinary order statistics and using a recurrence relation for single moments we obtain characterization of $p$ th order exponential distribution.
\end{abstract}

Keywords. Generalized order statistics; order statistics; record values; single moment; product moment; recurrence relations; $p$ th order exponential distribution; characterization.

MSC 2010: 62E10, 62E15.

\section{Introduction}

A random variable $X$ is said to have an $p$ th order exponential distribution if its probability density function $(p d f)$ is given by

$$
\begin{gathered}
f(x)=\left(a_{0}+a_{1} x+a_{2} x^{2}+\cdots+a_{p} x^{p}\right) e^{-\left(a_{0} x+a_{1} \frac{x^{2}}{2}+a_{2} \frac{x^{3}}{3}+\cdots+a_{p} \frac{x^{p+1}}{p+1}\right)} \\
x \geqslant 0, \quad a_{i}>0, \quad i=1,2, \ldots, p
\end{gathered}
$$

and the cumulative distribution function $(c d f)$ is

$$
F(x)=1-e^{-\left(a_{0} x+a_{1} \frac{x^{2}}{2}+a_{2} \frac{x^{3}}{3}+\cdots+a_{p} \frac{x^{p+1}}{p+1}\right)},
$$


where $p$ is some positive integer. For more details on this distribution and its application one may refer to Kamal (2010).

Kamps (1995) introduced the concept of generalized order statistics (gos) as follows: Let $X_{1}, X_{2}, \ldots$ be a sequence of independent and identically distributed (iid) random variables $(r \nu)$ with absolutely continuous $c d f \quad F(x)$ and $p d f \quad f(x), x \in(\alpha, \beta)$. Let $n \in N, n \geqslant 2, \tilde{m}=\left(m_{1}, m_{2}, \ldots, m_{n-1}\right) \in$ $\Re^{n-1}, k>0$, be the parameters such that

$$
\gamma_{r}=k+(n-r)+M_{r}>0 \text { for all } r \in\{1,2, \ldots, n-1\}
$$

where $M_{r}=\sum_{j=r}^{n-1} m_{j}$. Then $X(r, n, \tilde{m}, k), r=1,2, \ldots, n$ are called if their joint $p d f$ is given by

$$
k\left(\prod_{j=1}^{n-1} \gamma_{j}\right)\left[\prod_{i=1}^{n-1}\left\{1-F\left(x_{i}\right)\right\}^{m_{i}} f\left(x_{i}\right)\right]\left\{1-F\left(x_{n}\right)\right\}^{k-1} f\left(x_{n}\right)
$$

on the cone $F^{-1}(0)<x_{1} \leqslant \cdots \leqslant x_{n}<F^{-1}(1)$.

The model of gos contains as special cases, order statistics, record values, sequential order statistics, Stigler's order statistics.

Here we assume two cases:

Case I: $\quad m_{1}=m_{2}=\cdots=m_{n-1}=m$;

Case II: $\quad m_{i} \neq m_{j}, \gamma_{i} \neq \gamma_{j}, i, j=1,2, \ldots, n-1$.

For Case I, gos will be denoted as $X(r, n, m, k)$ with its $p d f$ (Kamps, 1995)

$$
f_{r}(x)=\frac{C_{r-1}}{(r-1) !}\{\bar{F}(x)\}^{\gamma_{r}-1} f(x)\left[g_{m}\{F(x)\}\right]^{r-1}
$$

and the joint $p d f$ of $X(r, n, m, k)$ and $X(s, n, m, k), 1 \leqslant r<s \leqslant n$, is

$$
\begin{aligned}
f_{r s}(x, y)= & \frac{C_{s-1}}{(r-1) !(s-r-1) !}\{\bar{F}(x)\}^{m}\left[g_{m}\{F(x)\}\right]^{r-1} \\
& \times\left[h_{m}\{F(y)\}-h_{m}\{F(x)\}\right]^{s-r-1}\{\bar{F}(y)\}^{\gamma_{s}-1} f(x) f(y), \quad x<y
\end{aligned}
$$

where 


$$
\begin{gathered}
\bar{F}(x)=1-F(x), \quad C_{r-1}=\prod_{i=1}^{r} \gamma_{i}, \\
h_{m}(x)= \begin{cases}-\frac{1}{m+1}(1-x)^{m+1}, & m \neq-1 \\
-\ln (1-x), & m=-1\end{cases}
\end{gathered}
$$

and

$$
g_{m}(x)=h_{m}(x)-h_{m}(0), \quad x \in[0,1) .
$$

For Case II, the $p d f$ of $X(r, n, \tilde{m}, k)$ is (Kamps and Cramer, 2001)

$$
f_{X(r, n, \tilde{m}, k)}(x)=C_{r-1} f(x) \sum_{i=1}^{r} a_{i}(r)\{\bar{F}(x)\}^{\gamma_{i}-1}
$$

and the joint $p d f$ of $X(r, n, \tilde{m}, k)$ and $X(s, n, \tilde{m}, k), 1 \leqslant r<s \leqslant n$, is

$$
\begin{aligned}
f_{X(r, n, \tilde{m}, k), X(s, n, \tilde{m}, k)}(x, y) & =C_{s-1} \sum_{i=r+1}^{s} a_{i}^{(r)}(s)\left\{\frac{\bar{F}(y)}{\bar{F}(x)}\right\}^{\gamma_{i}} \\
& \times\left[\sum_{i=1}^{r} a_{i}(r)\{\bar{F}(x)\}^{\gamma_{i}}\right] \frac{f(x)}{\bar{F}(x)} \cdot \frac{f(y)}{\bar{F}(y)}, \quad x<y
\end{aligned}
$$

where

$$
\begin{aligned}
a_{i}(r) & =\prod_{\substack{j=1 \\
j \neq i}}^{r} \frac{1}{\left(\gamma_{j}-\gamma_{i}\right)}, \quad \gamma_{j} \neq \gamma_{i}, \quad 1 \leqslant i \leqslant r \leqslant n, \\
a_{i}^{(r)}(s)= & \prod_{\substack{j=r+1 \\
j \neq i}}^{s} \frac{1}{\left(\gamma_{j}-\gamma_{i}\right)}, \quad \gamma_{j} \neq \gamma_{i}, \quad r+1 \leqslant i \leqslant s \leqslant n .
\end{aligned}
$$

Now since $\lim _{m \rightarrow-1} h_{m}(x)=\log \left(\frac{1}{1-x}\right)$, therefore, we will consider only the case $h_{m}(x)=-\frac{1}{m+1}(1-x)^{m+1}$ for all $m$, unless needed otherwise.

Several authors utilized the gos in their work, such as Kamps and Gather (1997), Keseling (1999), Cramer and Kamps (2000), Ahsanullah (2000), Pawlas and Szynal (2001), Ahmed and Fawzy (2003), Ahmed (2007), Khan, et al. (2007) among others. Kamps (1998) investigated the importance of 
recurrence relations of order statistics in characterization. Recurrence relations for moments of $k$-records were investigated, among others, by Grudzien and Szynal (1997), Pawlas and Szynal (1998, 1999).

In this paper, we establish recurrence relations for single and product moments of gos from $p$ th order exponential distribution. Result for order statistics and record values can be deduced as special cases from gos and characterization of this distribution has been obtained on using a recurrence relation for single moments.

\section{Recurrence Relations for Single Moments}

Note that for $p$ th order exponential distribution defined in (1)

$$
f(x)=\left(\sum_{u=0}^{p} a_{u} x^{u}\right) \bar{F}(x) .
$$

Recurrence relations for single moments of gos from $d f(2)$ can be derived in the following theorem.

Case I: $m_{i}=m_{g}=m, i, j=1,2, \ldots, n-1$.

Theorem 1. For the distribution given in (2) and $n \in N, m \in \Re, r=1$

$$
E\left\{X^{j}(1, n, m, k)\right\}=\sum_{u=0}^{p} \frac{\gamma_{1} a_{u}}{(j+u+1)} E\left\{X^{j+u+1}(1, n, m, k)\right\}
$$

and for $2 \leqslant r \leqslant n$

$$
\begin{aligned}
E\left\{X^{j}(r, n, m, k)\right\}= & \sum_{u=0}^{p} \frac{\gamma_{r} a_{u}}{(j+u+1)}\left[E\left\{X^{j+u+1}(r, n, m, k)\right\}\right. \\
& \left.-E\left\{X^{j+u+1}(r-1, n, m, k)\right\}\right]
\end{aligned}
$$

Proof. From (4) and (8), we have

$$
E\left\{X^{j}(r, n, m, k)\right\}=\sum_{u=0}^{p} a_{u} \frac{C_{r-1}}{(r-1) !} I_{r}(x),
$$

where

$$
I_{r}(x)=\int_{0}^{\infty} x^{j+u}\{\bar{F}(x)\}^{\gamma_{r}} g_{m}^{r-1}\{F(x)\} d x
$$


Integrating by parts treating $x^{j+u}$ for integration and the rest of the integrand for differentiation, we get

$$
\begin{aligned}
I_{r}(x)= & \frac{\gamma_{r}}{(j+u+1)} \int_{0}^{\infty} x^{j+u+1}\{\bar{F}(x)\}^{\gamma_{r}-1} f(x) g_{m}^{r-1}\{F(x)\} d x \\
& -\frac{(r-1)}{(j+u+1)} \int_{0}^{\infty} x^{j+u+1}\{\bar{F}(x)\}^{\gamma_{r}+m} f(x) g_{m}^{r-2}\{F(x)\} d x .
\end{aligned}
$$

Now substituting for $I_{1}(x)$ and $I_{r}(x)$ in equation (11), we drive the relations in (9) and (10).

Remark 1. Setting $m=0, k=1$ in Theorem 1, we obtain recurrence relations for single moments of order statistics of the $p$ th order exponential distribution in the form

$$
E\left(X_{1: n}^{j}\right)=n \sum_{u=0}^{p} \frac{a_{u}}{(j+u+1)} E\left(X_{1: n}^{j+u+1}\right)
$$

and

$$
E\left(X_{r: n}^{j}\right)=(n-r+1) \sum_{u=0}^{p} \frac{a_{u}}{(j+u+1)}\left\{E\left(X_{r: n}^{j+u+1}\right)-E\left(X_{r-1: n}^{j+u+1}\right)\right\} .
$$

These result was obtained by Kamal (2010).

Remark 2. Putting $m=-1, k \geqslant 1$ in (10), we get the recurrence relations for single moments of upper $k$-records of the $p$ th order exponential distribution in the form

$$
\begin{aligned}
E\left\{X^{j}(r, n,-1, k)\right\}= & k \sum_{u=0}^{p} \frac{a_{u}}{(j+u+1)}\left[E\left\{X^{j+u+1}(r, n,-1, k)\right\}\right. \\
& \left.+E\left\{X^{j+u+1}(r-1, n,-1, k)\right\}\right] .
\end{aligned}
$$

Case II: $m_{i} \neq m_{j}, \gamma_{i} \neq \gamma_{j}, i, j=1,2, \ldots, n-1$.

Theorem 2. For the distribution given in (2) and $2 \leqslant r \leqslant n, n \geqslant 2$ and $k=1,2, \ldots$

$$
E\left\{X^{j}(r, n, \tilde{m}, k)\right\}=\sum_{u=0}^{p} \frac{\gamma_{i} a_{u}}{(j+u+1)} E\left\{X^{j+u+1}(r, n, \tilde{m}, k)\right\}
$$


Proof. From (6) and (8), we have

$$
E\left\{X^{j}(r, n, m, k)\right\}=C_{r-1} \sum_{i=1}^{r} a_{i}(r) \sum_{u=0}^{p} a_{u} I_{r}(x),
$$

where

$$
I_{r}(x)=\int_{0}^{\infty} x^{j+u}\{\bar{F}(x)\}^{\gamma_{r}} g_{m}^{r-1}\{F(x)\} d x .
$$

Integrating by parts treating $x^{j+u}$ for integration and the rest of the integrand for differentiation, we get

$$
I_{r}(x)=\frac{\gamma_{i}}{(j+u+1)} \int_{0}^{\infty} x^{j+u+1}\{\bar{F}(x)\}^{\gamma_{r}-1} f(x) d x .
$$

Now substituting for $I_{r}(x)$ in equation (15), we drive the relations in (14).

\section{Recurrence Relations for Product Moments}

Making use of (8), we can drive recurrence relations for product moments of gos.

Case I: $m_{i}=m_{j}=m, i, j=1,2, \ldots, n-1$.

Theorem 3. For the given pth order exponential distribution in (2) and $n \geqslant 2, m \in \Re, 1 \leqslant r<r+1 \leqslant n$

$$
\begin{array}{r}
E\left\{X^{i}(r, n, m, k) X^{j}(r+1, n, m, k)\right\}=\gamma_{r+1} \sum_{u=0}^{p} \frac{a_{u}}{(j+u+1)}\left[E \left\{X^{i}(r, n, m, k)\right.\right. \\
\left.\left.X^{j+u+1}(r+1, n, m, k)\right\}-E\left\{X^{i+j+u+1}(r, n, m, k)\right\}\right]
\end{array}
$$

and for $1 \leqslant r<s \leqslant n, s-r \geqslant 2$, and $i, j \geqslant 0$

$$
\begin{aligned}
& E\left\{X^{i}(r, n, m, k) X^{j}(s, n, m, k)\right\}=\gamma_{r} \sum_{u=0}^{p} \frac{a_{u}}{(j+u+1)}\left[E \left\{X^{i}(r, n, m, k)\right.\right. \\
&\left.\left.X^{j+u+1}(s, n, m, k)\right\}-E\left\{X^{i}(r, n, m, k) X^{j+u+1}(s-1, n, m, k)\right\}\right] .
\end{aligned}
$$


Proof. From (5), we have

$$
\begin{aligned}
E\left\{X^{i}(r, n, m, k) X^{j}(s, n, m, k)\right\}= & \frac{C_{s-1}}{(r-1) !(s-r-1) !} \int_{0}^{\infty} x^{i}\{\bar{F}(x)\}^{m} \\
& \times f(x) g_{m}^{r-1}\{F(x)\} I(x) d x
\end{aligned}
$$

where

$$
\begin{aligned}
I(x) & =\int_{x}^{\infty} y^{j}\left[h_{m}\{F(y)\}-h_{m}\{F(x)\}\right]^{s-r-1}\{\bar{F}(y)\}^{\gamma_{s}-1} f(y) d y \\
& =\sum_{u=0}^{p} a_{u} \int_{x}^{\infty} y^{j+u}\left[h_{m}\{F(y)\}-h_{m}\{F(x)\}\right]^{s-r-1}\{\bar{F}(y)\}^{\gamma_{s}} d y
\end{aligned}
$$

upon using the relation in (8). Integrating now by parts treating $y^{j+u}$ for integration and the rest of the integrand for differentiation, we obtain when $s=r+1$ that

$$
\begin{aligned}
I(x)= & \sum_{u=0}^{p} \frac{a_{u}}{(j+u+1)}\left[-x^{j+u+1}\{\bar{F}(x)\}^{\gamma_{r+1}}+\gamma_{r+1}\right. \\
& \left.\times \int_{x}^{\infty} y^{j+u+1}\{\bar{F}(y)\}^{\gamma_{r+1}-1} f(y) d y\right]
\end{aligned}
$$

and when $s>r+1$ that

$$
\begin{aligned}
I(x)= & \sum_{u=0}^{p} \frac{a_{u}}{(j+u+1)}\left\{\gamma_{r} \int_{x}^{\infty} y^{j+u+1}\left[h_{m}\{F(y)\}-h_{m}\{F(x)\}\right]^{s-r-1}\right. \\
& \{\bar{F}(y)\}^{\gamma_{s}-1} f(y) d y-\frac{(s-r-1)}{(j+u+1)} \\
& \left.\int_{x}^{\infty} y^{j+u+1}\left[h_{m}\{F(y)\}-h_{m}\{F(x)\}\right]^{s-r-2}\{\bar{F}(y)\}^{\gamma_{s}+m} f(y) d y\right\} .
\end{aligned}
$$

Upon substituting the above expressions for $I(x)$ in (18), we have, after simplifications, the recurrence relations (16) and (17).

Remark 3. Setting $m=0, k=1$ in (16) and (17), we obtain recurrence relations for product moments of order statistics of the $p$ th order exponential distribution in the form

$$
E\left(X_{r, r+1: n}^{(i, j)}\right)=(n-r) \sum_{u=0}^{p} \frac{a_{u}}{(j+u+1)}\left\{E\left(X_{r, r+1: n}^{(i, j+u+1)}\right)-E\left(X_{r: n}^{(i+j+u+1)}\right)\right\}
$$


and

$$
E\left(X_{r, s: n}^{(i, j)}\right)=(n-s+1) \sum_{u=0}^{p} \frac{a_{u}}{(j+u+1)}\left\{E\left(X_{r, s: n}^{(i, j+u+1)}\right)-E\left(X_{r, s-1: n}^{(i, j+u+1}\right)\right\}
$$

these result was obtained by Kamal (2010).

Remark 4. Putting $m=-1, k \geqslant 1$ in (17), we get the recurrence relations for product moments of upper $k$-records of the $p$ th order exponential distribution in the form

$$
\begin{aligned}
& E\left\{X^{i}(r, n,-1, k) X^{j}(s, n,-1, k)\right\}=k \sum_{u=0}^{p} \frac{a_{u}}{(j+u+1)}\left[E \left\{X^{i}(r, n,-1, k)\right.\right. \\
&\left.\left.X^{j+u+1}(s, n,-1, k)\right\}-E\left\{X^{i}(r, n,-1, k) X^{j+u+1}(s-1, n,-1, k)\right\}\right] .
\end{aligned}
$$

Case II: $m_{i} \neq m_{j}, \gamma_{i} \neq \gamma_{j}, i, j=1,2, \ldots, n-1$.

Theorem 4. For the given pth order exponential distribution in (2) and for $1 \leqslant r<s \leqslant n-1, n \geqslant 2$ and $k=1,2, \ldots$

$$
\begin{array}{r}
E\left\{X^{i}(r, n, \tilde{m}, k) X^{j}(s, n, \tilde{m}, k)\right\}=\sum_{u=0}^{p} \frac{a_{u}}{(j+u+1)}\left[\gamma _ { i } E \left\{X^{i}(r, n, \tilde{m}, k)\right.\right. \\
\left.\left.X^{j+u+1}(s, n, \tilde{m}, k)\right\}-E\left\{X^{i+j+u+1}(r, n, \tilde{m}, k)\right\}\right]
\end{array}
$$

Proof. From (7), we have

$$
\begin{aligned}
E\left\{X^{i}(r, n, \tilde{m}, k) X^{j}(s, n, \tilde{m}, k)\right\}= & C_{s-1} \int_{0}^{\infty} x^{i} \sum_{i=r+1}^{s} a_{i}^{(r)}(s)\left\{\frac{1}{\bar{F}(x)}\right\}^{\gamma_{i}} \\
& {\left[\sum_{i=1}^{r} a_{i}(r)\{\bar{F}(x)\}^{\gamma_{i}}\right] \frac{f(x)}{\bar{F}(x)} I(x) d x }
\end{aligned}
$$

where

$$
\begin{aligned}
I(x) & =\int_{x}^{\infty} y^{j}\{\bar{F}(y)\}^{\gamma_{i}} \frac{f(x)}{\bar{F}(x)} d y \\
& =\sum_{u=0}^{p} a_{u} \int_{x}^{\infty} y^{j+u}\{\bar{F}(y)\}^{\gamma_{i}} d y
\end{aligned}
$$


upon using the relation in (8). Integrating now by parts treating $y^{j+u}$ for integration and the rest of the integrand for differentiation

$$
\begin{aligned}
I(x)= & \sum_{u=0}^{p} \frac{a_{u}}{(j+u+1)}\left[-x^{j+u+1}\{\bar{F}(x)\}^{\gamma_{i}}\right. \\
& \left.+\gamma_{i} \int_{x}^{\infty} y^{j+u+1}\{\bar{F}(y)\}^{\gamma_{i}-1} f(y) d y\right] .
\end{aligned}
$$

Upon substituting the above expressions for $I(x)$ in $(24)$, we have, after simplifications, the recurrence relations (23).

\section{Characterization}

Theorem 5. Let $X$ be a non-negative random variable having an absolutely continuous distribution function $F(x)$ with $F(0)=0$ and $0<F(x)<1$ for all $x>0$, then

$$
\begin{aligned}
E\left\{X^{j}(r, n, m, k)\right\}= & \sum_{u=0}^{p} \frac{\gamma_{r} a_{u}}{(j+u+1)} E\left\{X^{j+u+1}(r, n, m, k)\right\} \\
& -\sum_{u=0}^{p} \frac{\gamma_{r} a_{u}}{(j+u+1)} E\left\{X^{j+u+1}(r-1, n, m, k)\right\}
\end{aligned}
$$

if and only if

$$
F(x)=1-e^{-\left(a_{0} x+a_{1} \frac{x^{2}}{2}+a_{2} \frac{x^{3}}{3}+\cdots+a_{p} \frac{x^{p+1}}{p+1}\right)} .
$$

Proof. The necessary part follows immediately from equation (10). On the other hand if the recurrence relation in equation (26) is satisfied, then on using equation (4), we have

$$
\begin{aligned}
& \frac{C_{r-1}}{(r-1) !} \int_{0}^{\infty} x^{j}\{\bar{F}(x)\}^{\gamma_{r}-1} f(x) g_{m}^{r-1}\{F(x)\} d x= \\
& \quad \frac{C_{r-1}}{(r-1) !} \sum_{u=0}^{p} \frac{\gamma_{r} a_{u}}{(j+u+1)} \int_{0}^{\infty} x^{j+u+1}\{\bar{F}(x)\}^{\gamma_{r}-1} f(x) g_{m}^{r-1}\{F(x)\} d x \\
& \quad-\frac{(r-1) C_{r-1}}{(r-1) !} \sum_{u=0}^{p} \frac{\gamma_{r} a_{u}}{(j+u+1)} \int_{0}^{\infty} x^{j}\{\bar{F}(x)\}^{\gamma_{r}+m} f(x) g_{m}^{r-2}\{F(x)\} d x
\end{aligned}
$$


Integrating the second integral on the right hand side of equation (27), by parts, we get

$$
\begin{aligned}
& \frac{C_{r-1}}{(r-1) !} \int_{0}^{\infty} x^{j}\{\bar{F}(x)\}^{\gamma_{r}-1} f(x) g_{m}^{r-1}\{F(x)\} d x= \\
& \frac{\gamma_{r} C_{r-1}}{(r-1) !} \sum_{u=0}^{p} \frac{a_{u}}{(j+u+1)} \int_{0}^{\infty} x^{j+u+1}\{\bar{F}(x)\}^{\gamma_{r}-1} f(x) g_{m}^{r-1}\{F(x)\} d x \\
& +\frac{C_{r-1}}{(r-1) !} \sum_{u=0}^{p} \frac{(j+u+1) a_{u}}{(j+u+1)} \int_{0}^{\infty} x^{j+u}\{\bar{F}(x)\}^{\gamma_{r}} g_{m}^{r-1}\{F(x)\} d x \\
& -\frac{\gamma_{r} C_{r-1}}{(r-1) !} \sum_{u=0}^{p} \frac{a_{u}}{(j+u+1)} \int_{0}^{\infty} x^{j+u+1}\{\bar{F}(x)\}^{\gamma_{r}-1} f(x) g_{m}^{r-1}\{F(x)\} d x
\end{aligned}
$$

which reduces to

$$
\frac{C_{r-1}}{(r-1) !} \int_{0}^{\infty} x^{j}\{\bar{F}(x)\}^{\gamma_{r}-1} g_{m}^{r-1}\{F(x)\} d x\left[f(x)-\left(\sum_{u=0}^{p} a_{u} x^{u}\right)\{\bar{F}(x)\}\right]=0
$$

Now applying a generalization of the Müntz-Szász Theorem (Hwang and Lin, 1984) to equation (28), we get

$$
\frac{f(x)}{\bar{F}(x)}=\sum_{u=0}^{p} a_{u} x^{u}
$$

which prove that

$$
F(x)=1-e^{-\left(a_{0} x+a_{1} \frac{x^{2}}{2}+a_{2} \frac{x^{3}}{3}+\cdots+a_{p} \frac{x^{p+1}}{p+1}\right)}, \quad x \geqslant 0 .
$$

\section{Remarks}

1. Putting $p=0, a_{0}=1$ and $a_{u}=0 \quad u \geqslant 1$ in (13) and (22), we get the results of Joshi $(1978,1982)$.

2. Setting $p=1, a_{0}=0, a_{1}=0$ and $a_{u}=0 \quad u \geqslant 2$ in (13) and (22), we get the results Rayleigh distribution.

3. Setting $p=1, a_{0}=\lambda, a_{1}=\nu$ and $a_{u}=0 \quad u \geqslant 2$ in (13) and (22), we get the results of Balakrishnan and Malik (1986b) and of Mohie El-Din et al. (1997). 


\section{Conclusion}

This paper deals with the generalized order statistics from the $p$ th order exponential distribution. Some recurrence relations between the single and product moments are derived. Characterization of the $p$ th order exponential distribution by using recurrence relation of single moment. Special cases are also deduced.

\section{References}

Ahmad, A.A. and Fawzy, M., (2003). Recurrence relations for single moments of generalized order statistics from doubly truncated distribution, J. Statist. Plann. Inference, 117, 241-249.

Ahmad, A.A. (2007). Relations for single and product moments of generalized order statistics from doubly truncated Burr type XII distribution, J. Egypt. Math. Soc., 15, 117-128.

Ahsanullah, M., (2000). Generalized order statistics from exponential distribution, J. Statist. Plann. Inference, 85, 85-91.

Balakrishnan, N. and Malik, H.J. (1986). Order statistics from linear exponential distribution. Part I: Increasing hazard rate case. Comm. Statist. Theory Method, 15, 179-203.

Cramer, E. and Kamps, U. (2000). Relations for expectations of functions of generalized order statistics, J. Statist. Plann. Inference, 89, 79-89.

Grunzien, Z. and Szynal, D. (1997). Characterization of uniform and exponential distributions via moments of the record values with random indices, Appl. Statist. Sci., 5, 259-266.

Hwang, J.S. and Lin, G.D. (1984). On a generalized moments problem II. Proc. Amer. Math. Soc., 91, 577-580.

Joshi, P.C. (1978). Recurrence relation between moments of order statistics from exponential and truncated exponential distributions, Sankhy $\bar{a}$ Ser. B, 39, 362-371.

Joshi, P.C. (1982). A note on mixed moments of order statistics from exponential and truncated exponential distributions, J. Statist. Plann. Inference, 6, 13-16.

Kamal N. (2010). Recurrence relations for single and product moments of ordinary order statistics from $p^{\text {th }}$ order exponential distribution, Int. Math. Forum, 5, 1653-1662.

Kamps, U. and Cramer, E. (2001). On distributions of generalized order statistics, Statistics, 35, 269-280.

Kamps, U. and Gather, U. (1997). Characteristic property of generalized order statistics for exponential distribution, Appl. Math. (Warsaw), 24, 383-391. 
Kamps, U. (1995). A Concept of Generalized Order Statistics. B.G. Teubner Stuttgart.

Kamps, U. (1998). Characterizations of distributions by recurrence relations and identities for moments of order statistics. In: Balakrishnan, N. and. Rao, C. R eds., Hand book of statistics, 16, Order Statistics, Theory and Methods, Amsterdam: North-Holand, 16, 291-311.

Keseling, C. (1999). Conditional distributions of generalized order statistics and some characterizations, Metrika, 49, 27-40.

Khan, R.U.; Anwar, Z. and Ather, H., (2007). Recurrence relations for single and product moments of generalized order statistics from doubly truncated weibull distribution, Aligarh J. Statist., 27, 69-79.

Molie El-Din, M.M.; Mahmoud, M.A.W.; Abu-Youssef, S.E. and Sultan, K.S. (1997). Order statistics from doubly truncated linear exponential distribution and its characterizations Comm. Statist.-Simul. Comput., 26, 281-290.

Pawlas, P. and Szynal, D. (1998). Relations for single and product moments of $k$-th record values from exponential and Gumbel distributions, J. Appl. Statist. Sci., 7, 53-61.

Pawlas, P. and Szynal, D. (1999). Recurrence relations for single and product moments of $k$-th record values from Pareto, generalized Pareto and Burr distributions, Comm. Statist. Theory Methods, 28, 1699-1709.

Pawlas, P. and Szynal, D. (2001). Recurrence relations for single and product moments of generalized order statistics from Pareto, generalized Pareto, and Burr distributions, Comm. Statist. Theory Methods, 30, 739-746.

\section{Devendra Kumar}

Department of Statistics and Operations Research,

Aligarh Muslim University,

Aligarh-202 002, India.

email:devendrastats@gmail.com 